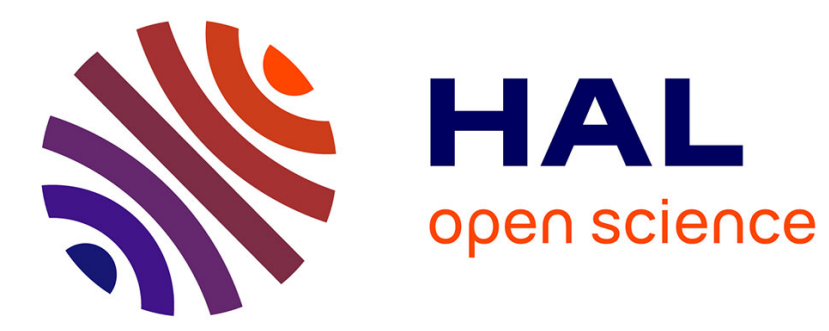

\title{
Le débat sur la représentation des saints à San Lorenzo del Escorial après le concile de Trente
}

Sylvène Edouard

\section{To cite this version:}

Sylvène Edouard. Le débat sur la représentation des saints à San Lorenzo del Escorial après le concile de Trente. Revue d'Histoire ecclésiastique , 2006, 101.1, pp.88-116. halshs-00068953

\section{HAL Id: halshs-00068953 \\ https://shs.hal.science/halshs-00068953}

Submitted on 15 May 2006

HAL is a multi-disciplinary open access archive for the deposit and dissemination of scientific research documents, whether they are published or not. The documents may come from teaching and research institutions in France or abroad, or from public or private research centers.
L'archive ouverte pluridisciplinaire HAL, est destinée au dépôt et à la diffusion de documents scientifiques de niveau recherche, publiés ou non, émanant des établissements d'enseignement et de recherche français ou étrangers, des laboratoires publics ou privés. 
Sylvène Edouard,

Maître de Conférences à l'Université Lyon 3

Membre de RESEA (LAHRA-UMR CNRS 5190)

\section{Le débat sur la représentation des saints à San Lorenzo del Escorial après le concile de Trente}

L'année 1563 fut marquée par le début de la construction du Palais-Monastère de l'Escorial par Philippe II et par la clôture du concile de Trente. L'Escorial devint ensuite un modèle de la réforme catholique en répondant à la controverse protestante par la matérialisation des décrets réformateurs du concile. De ce point de vue, le programme pictural des saints rend compte des réponses apportées par l'art pour défendre la « citadelle assiégée ». Les choix adoptés révèlent les règles nouvelles de cet art concernant l'image de religion. Bible des illettrés, selon la formule grégorienne, l'image est, par son langage, le support d'un enseignement et d'une dévotion. «... En l'image gît une puissance de religion et [...] son message, comme il se donne, est nourriture de la ferveur commune ${ }^{1}$. L'image s'exprime par des modèles illustrant plusieurs de ses vocations pour favoriser le culte et la dévotion, sur le mode de l'apologie, de l'édification spirituelle, de la pédagogie mais aussi en affirmant le dogme.

L'élaboration du programme hagiographique pour l'Escorial est un témoignage de l'application presque immédiate du décret tridentin sur la liturgie et les saintes images, dans un temps court et très proche de la clôture du concile de Trente, jusque dans les années 1580 . Les travaux consacrés à Philippe II en lien avec le concile de Trente sont importants, qu'il s'agisse des modalités de réception des décrets ou de leur impact sur la réforme du clergé en Espagne $^{2}$. La liturgie post-tridentine, en Espagne, a également fait l'objet d'études, mais il reste la question des saints, dont l'importance n'est autre que celle de la révision des hagiographies dans la liturgie et $l^{\prime} \operatorname{art}^{3}$. Au cœur de dévotions anciennes, certains saints de la

\footnotetext{
${ }^{1}$ Alphonse DUPRONT, Du Sacré. Croisades et pèlerinages. Images et langages, Paris, Gallimard, 1987, p. 100.

${ }^{2}$ Ignasi FERNÁNDEZ TERRICABRAS, Philippe II et la Contre-Réforme. L'Église espagnole à l'heure du concile de Trente, Paris, Publisud, 2001. Il est également l'auteur de plusieurs publications sur la réforme des ordres religieux et des chapitres et sur les visites pastorales. Peuvent être également consultés des ouvrages généraux, ceux de Vicente Cadenas y Vicent et de Ricardo García-Villoslada, les monographies régionales de Carlos Alonso sur l'Aragon, de José Luis González Novalín sur Oviedo ; sur les relations ad limina avec Vicente Cárcel Ortí, sur les ordres avec Manuel de Castro, OFM, pour les franciscains, Teófanes Egido pour sainte Thérèse, ainsi que José García Oro pour les carmélites, Ernesto Zaragoza Pascual sur les bénédictins et les nombreux travaux de José Ignacio Tellechea Idígoras.

${ }^{3}$ On peut consulter sur le sujet de l'art religieux post-tridentin à San Lorenzo del Escorial, l'article de synthèse de Fernando COLLAR DE CÁCERES, «Arte y rigor religioso. Españoles e italianos en el ornato de los retablos del Escorial (Altares comunes y altares de reliquias) » dans Felipe II y el arte de su tiempo, Madrid, Fundación Argentaria, 1998, pp. 79-119. Les ouvrages les plus complets sont ceux de R. MULCAHY, A Mayor Gloria del Rey. La décoración de la Real Basílica del Monasterio de El Escorial, Madrid, 1992 et de Cornelia von DER OSTEN SACKEN, El Escorial. Estudio iconológico, Madrid, Xarait, 1984. Fernando CHECA apporte également une contribution intéressante dans Felipe II. Mecenas de las artes, Madrid, Nerea, 1992.
} 
tradition, tenant plus de la fable que de l'histoire sainte, auraient dû disparaître, conformément au décret conciliaire. Il n'en fut rien et cela montre les limites que la tradition imposa à la réforme. Quels furent les intentions nouvelles de la représentation hagiographique à l'Escorial, le lexique de son discours apologétique et ses enjeux dogmatiques pour observer le décret tridentin sur les saintes images et quels furent en outre ses procédés didactiques portant le fidèle sur la voie d'un cheminement dévotionnel, voie inscrite dans l'espace des saints à l'Escorial?

En 1566, Ambrosio de Morales, « antiquaire » et spécialiste en hagiographie auprès de Philippe II, remit à ce dernier un mémoire portant sur la réforme du contenu des leçons consacrées aux saints. Il établissait des règles qui furent suivies par les artistes italiens et espagnols ayant représenté, durant ces décennies, une soixantaine de saints, essentiellement pour l'église, le couvent et le collège. Dès 1566, les règles, qui fixèrent le principe de l'énoncé iconographique des sujets religieux de même que celui des missels, bréviaires, lectionnaires et autres, furent au nombre de trois. Ces règles furent tout d'abord le recours aux faits authentiques, auxquels il fallait donner une noblesse limitée à la vraisemblance et cela dans un langage facilement accessible. En trois mots : véracité, dignité et simplicité.

\section{La règle de la véracité pour une dévotion sans abus}

Les saints avaient été, à plus d'un titre, sujets à controverse. Ils étaient vénérés comme médiateurs et comme guérisseurs à travers leurs ossements et attributs précieux. Or, le trafic déjà ancien des reliques en avait considérablement augmenté le nombre et remis en cause l'authenticité. En 1543, dans son Traité des Reliques, Jean Calvin reprochait non seulement aux reliques de susciter "une superstition païenne» mais aussi d'introduire "pollution et ordure » dans la pratique religieuse ${ }^{4}$. La doctrine réformée calviniste niait le rôle médiateur des saints et considérait leurs images comme autant d'idoles qui n'ont pas leur place dans l'église, où la prédication ne doit se faire que par la parole. En quelques décennies, une partie de l'Europe gagnée par ce discours, renonçait au culte des saints, brisant images, statues et reliques, phénomène atteignant son paroxysme en 1562 en France et en 1566 aux Pays-Bas à travers l'iconoclasme calviniste 5 . En réponse, le concile de Trente émit lors de sa vingtcinquième session, en décembre 1563, un décret "sur l'intercession des saints et leur invocation », rappelant « les honneurs dus aux reliques et le légitime usage des images ${ }^{6}$. La réponse n'était pas nouvelle, reprenant les déclarations de Nicée II (787) sur la légitimité des images et celles de Latran IV (1215) sur le contrôle des excès et la vérification de l'authenticité, afin que «ceux qui viennent dans leurs églises en vue de vénérer des reliques [ne] soient trompés par de vaines fictions ou de faux documents ${ }^{7}$. Malgré ce décret de Latran, les légendes des saints perdurèrent et firent florès comme La légende dorée de Jacques de Voragine au XIIIe siècle, les évangiles apocryphes sur les enfances de Marie et de Jésus, le livre attribué à Abdias sur les Douze Apôtres et bien d'autres Actes encore. Dans son important Traité des saintes images de 1570, le professeur de Louvain, Johannes Molanus

\footnotetext{
${ }^{4}$ Jean CALVIN, Traité des Reliques, par Irena Backus, Genève, Labor et Fides, 2000, p. 73.

${ }^{5}$ Olivier CHRISTIN, Une révolution symbolique : l'iconoclasme huguenot et la reconstruction catholique, Paris, éd. de Minuit, 1991, et Stéphane BOIRON, La controverse née de la querelle des Reliques à l'époque du concile de Trente (1500-1640), Paris, PUF, 1989. Voir également l'introduction à Johannes MOLANUS, Traité des saintes images. Introduction, traduction, notes et index par François Boespflug, Olivier Christin, Benoît Tassel, Paris, éd. du Cerf, 1996.

${ }^{6}$ « Décret sur l'invocation, la vénération et les reliques des saints, et sur les saintes images », dans Les conciles acuméniques, Tome II-2, Les décrets (Trente à Vatican II), Paris, éd. du Cerf, 1994, pp. 774-776.

${ }^{7}$ Chapitre 62 de Latran IV, dans Les conciles ecuméniques, Tome II-2, op. cit., p. 263.
} 
souligne d'ailleurs que si ces ouvrages furent condamnés par l'Inquisition romaine en 1559, ils ne figurèrent pas pour autant dans l'Index ${ }^{8}$.

Que penser alors de la règle de la véracité qui fut l'un des principaux enjeux de la dévotion sans abus, pure de toute fable?

[...] qu'on expose aucune image porteuse d'une fausse doctrine et pouvant être l'occasion d'une erreur dangereuse pour les gens simples 9 .

Le principe de la véracité énoncé au concile de Trente fut l'un des moins explicites, car il omettait de préciser ce qu'il n'était plus convenu de croire dans la tradition hagiographique. La question de l'authenticité était déjà ancienne et amplement débattue. Si aucune théorie ne fut réellement approfondie tandis qu'en Orient faisait rage la crise iconoclaste au VIIIe siècle, certains arguments parvinrent un siècle plus tard en Occident et initièrent une nouvelle tendance limitant les dévotions aux saints. L’Église, dans sa volonté de régler le rapport de l'individu au sacré, perçut alors l'ambiguïté consistant à soutenir la foi des fidèles au risque d'entretenir superstitions et hérésies. Le droit canon, sur une longue durée s'étendant au moins jusqu'au XIIIe siècle, fixa les conditions de l'authenticité (contre les faux martyrs) et des usages des reliques des saints. Ce fut à Latran, en 1215, qu'un décret conciliaire confiait au Saint-Siège le contrôle des dévotions aux reliques et traitait d'une façon générale du culte des nouveaux saints et de l'authentification de leurs reliques.

$\mathrm{Au}$ XVIe siècle, la question de l'authenticité renvoyait à l'hagiographie héritée du bas Moyen Âge (dès le XIe siècle), moins portée sur les vertus des saints que sur leurs miracles. La défense des saints et de leurs reliques devait passer par un examen de leurs vies, ce que ne manquèrent pas d'entreprendre nombre d' "antiquaires » versés dans l'hagiographie. En Espagne, Juan Luis Vivés et le théologien Melchior Cano avaient souligné les fraudes et les mensonges en matière d'hagiographie ${ }^{10}$. Ambrosio de Morales, commis par Philippe II en 1572 pour repérer les reliques dans les églises et les monastères de l'ancien royaume asturoléonais, se montra soucieux de leur authenticité et semble avoir lui-même composé un traité sur la question, intitulé Discurso de los buenos testimonios que puede haver para tener una Reliquia por cierta $^{11}$. Mandaté par le roi pour réformer la lecture des saints dans les missels et les bréviaires (réforme tridentine des livres liturgiques), Morales avait été confronté au problème de l'authenticité de la tradition hagiographique. Pressé par les circonstances de l'après-concile, Morales soulignait combien il était alors difficile de parler des saints de façon certaine et vérifiée. Il déplorait l'insuffisance des écrits anciens en la matière, ceux « des saints et d'autres auteurs anciens qui purent beaucoup écrire à ce propos et nous aider en cela mais qui ne laissèrent rien ou si peu $»^{12}$. Il considérait les hagiographies plus récentes peu

\footnotetext{
8 Johannes MOLANUS, Traité des saintes images. Introduction, traduction, notes et index par François Bloespflug, Olivier Christin, Benoît Tassel, Paris, éd. du Cerf, 1996, p. 204-205.

${ }^{9}$ Voir note 6.

${ }^{10}$ Voir Melchior CANO, De locis theologicis Libri duodecim, Salamanque, Mathias Gastius, 1563, et Juan Luis VIVÉS, De Disciplinis libri XX, Anvers, Michael Hillenius, 1531.

${ }^{11}$ Ce manuscrit, cité par Morales dans son enquête, ne figure dans aucune bibliographie ancienne. Sur l'enquête de Morales, voir Sylvène ÉDOUARD, «Enquête hagiographique et mythification historique. Le « saint voyage » d'Ambrosio de Morales (1572) », dans Mélanges de la Casa de Velázquez, Tome 33-2, 2003, pp. 33-60, ainsi que Ambrosio de MORALES, Viage de Ambrosio de Morales por orden del Rey D. Phelipe II a los Reynos de León y Galicia y Principado de Asturias. Para reconocer las Reliquias de Santos, Sepulcros Reales y Libros manuscritos de las Cathedrales y Monasterios, Madrid, 1765, p. 206.

12 Ambrosio de MORALES, Copia de los apuntamientos que tubo Ambrosio de Morales sobre los liçionarios para la orden de Su Magestad y de la repuesta que Su Magestad mando hazer a ellos. En Março 1566, «Lo que paresce se deve advertir para hazer con acertamientos las leciones de los santos... », Archivo general de Simancas [AGS], Casas y Sitios Reales, dossier 258, fol. 225. Les citations sont traduites par l'auteur, avec
} 
fiables à l'exception des nombreux volumes, en réalité discutables, de Las Vidas y hechos de los santos de l'évêque de Vérone Aloïs Lippomano, dont il saluait l'esprit critique et l'érudition, enrichie au contact de la bibliothèque vaticane ${ }^{13}$. Il était devenu impératif de réviser les vies des saints et le martyrologe romain. En Espagne, le frère franciscain Martin de Lilio publia chez Juan de Brocar, à Alcalá, un Flos sanctorum en 1558 tandis que le Père jésuite Pedro de Ribadeneyra publiait ses deux parties du Flos sanctorum, o Libro de las Vidas de los Santos à Madrid, entre 1599 et 1601. Cette dernière compilation dépassait celles qui avaient été entreprises en Espagne à la même époque, comme celle d'Alonso de Villegas, chapelain à Tolède, qui publia en six parties, de 1578 à 1603, un Flos sanctorum dont les trois premières parties étaient consacrées aux saints d'après les vies des saints de Lippomano, Sirius et Voragine! Les exemplaires de la troisième partie, concernant les vies des saints « extravagantes », furent d'ailleurs confisqués par l'Inquisition en 1589. Les exemplaires sauvés de la destruction le furent grâce à certaines bibliothèques conventuelles qui dissimulèrent leurs exemplaires en maquillant la page de titre ${ }^{14}$. Malgré ce succès de la tradition, la révision des hagiographies était bien amorcée avec la première rédaction officielle du martyrologe romain du cardinal Cesare Baronius (1538-1607) en 1584. L'érudition catholique avait ainsi commencé à rompre avec la légende dorée pour retrouver les récits originaux. L'œuvre critique poursuivit son essor, dès 1643, avec les Acta Sanctorum de Jean Bolland ${ }^{15}$.

Dans son mémoire commandé par le roi, Ambrosio de Morales énonçait quelques principes de précaution pour écrire « de quelque manière les vies des saints et raconter leurs actes $\gg{ }^{16}$. En mars 1566, il faisait parvenir ses avertissements sur les lectionnaires des saints et de Notre Dame pour l'ordre de saint Jérôme. D'après le décret conciliaire, missel, bréviaire et catéchisme devaient être communs à tous les ordres. Philippe II obtint néanmoins de Pie V le droit de procéder à des modifications. Les hiéronymites de l'Escorial obtinrent même le privilège de monopole de la distribution en Espagne et aux Indes des livres de liturgie ${ }^{17}$. Dans le travail de réforme des livres liturgiques de l'ordre entamé par le Père général et Ambrosio de Morales, le premier souci avait été de vérifier les récits hagiographiques pour ne retenir que les plus certifiés. Les vies des saints exposées dans les leçons devaient être tirées des auteurs les plus approuvés, cela impliquant la remise en question de certaines traditions anciennes de l'Église. Morales insiste, à juste titre, sur les efforts également anciens de l'Église pour réviser la tradition de saints faisant l'objet d'une grande dévotion. Philippe II souligna, comme suit, les passages importants du texte de Morales et les commenta par des annotations en marge, placées ici entre crochets :

\footnotetext{
l'aide de Margarita Torrione que je remercie, et elles sont reproduites en note : « los sanctos y otros escriptores antíguos que pudieron scrivír desto y ayudarnos bien en ello o no dexaron nada o muy poco scripto. »

${ }^{13}$ D'après L'Histoire des auteurs sacrés et ecclésiastiques du R.P. Dom Rémy CEILLIER [Paris, Louis Vivès, 1869], Aloïs Lippomano aurait repris, en l'abrégeant, le martyrologe d'Adon, archevêque de Vienne en 860. Il s'agit d'une édition vénitienne de 1554, reprenant donc les vies semi-légendaires des saints rédigées par Adon dont le martyrologe connut un grand succès puisqu'il fut de nouveau édité en version complète par Mosander en 1581 à Cologne et enfin par Rosweyde à Anvers en 1613. Entre 1570 et 1575, Laurent SURIUS, chartreux de Cologne, publia six volumes dédiés à Pie V des Vitae sanctorum ab Aloysio Lippomano olim conscriptae, desquels il élimina les vies des saints les moins attestées.

${ }^{14}$ Antonio PALAU Y DULCET, Manuel del librero hispanoamericano, T. 27, Barcelona, 1976, $\mathrm{n}^{\circ} 369145$.

${ }^{15}$ Nicole HERRMANN-MASCARD, Les Reliques des saints : formation coutumière d'un droit, Paris, Klincksieck, 1975.

${ }^{16}$ MORALES, « lo que paresce se deve advertir... », op. cit. : « de qualquiera manera las vidas de los sanctos y contar sus hechos .»

${ }^{17}$ Christian PÉLIGRY, «El monasterio de San Lorenzo de El Escorial y la difusión de los libros liturgicos en España (1573-1615) », Primeras jornadas de Bibliografía. Mayo 1976, Madrid, Fundación Universitaria Española, 1977, pp. 465-473.
} 
Comme, depuis longtemps, en cette matière et en tant d'autres, il y eut toujours de bons et notables écrivains mais que ceux qui ne l'étaient pas ont prévalu dans ce domaine par la simplicité de quelques mols et pieux chrétiens, donnant plus de prix à ces choses, leur enseignant et les recommandant en d'autres encore plus saintes et pleines de dévotion et cela montre bien le sentiment qu'a toujours eu à ce propos la sainte église romaine depuis de nombreux siècles qu'il lui a paru nécessaire de remédier à ce mal, au moyen de graves décrets ordonnant que les écrits sur les actes des saints et les histoires des martyrs dussent exclure et rejeter comme étant apocryphes bien qu'elles fussent très anciennes au sein de l'église [les choses que l'église, par ses décrets, tient pour sûres, qu'elles soient exclues et rejetées. Si, dans les lectionnaires dont il est fait usage, il se trouve de ces choses, il est très juste de les retirer et de les remplacer par d'autres plus approuvées] et diffusées de main en main - il est prouvé, depuis de nombreux siècles, que l'église les conserve en grande partie - et lues à haute voix, augmentées parfois d'autres nouvelles choses qui ressemblent aux pieuses choses évoquées plus haut. Tout cela doit être abandonné dans les leçons pour en trouver de plus sûres chez les auteurs anciens, le choix devant être fait avec prudence. ${ }^{18}$

L'affaire était délicate car nombre de traditions apocryphes faisaient l'objet de croyances et de dévotions, lues et commentées lors des offices. Or, la véracité était devenue un enjeu important de l'orthodoxie catholique fixant un modèle d'imitation pour les fidèles afin de contribuer à la fermeté de la foi. Respectueux du décret conciliaire, Philippe II et son historiographe étaient prêts à bouleverser des croyances anciennes pour rétablir les vies des saints dans leur vérité mais à la seule condition de les modifier pour des faits certifiés :

[...] Il convient de regarder avec attention et d'agir avec beaucoup de diligence pour que tout ce qui sera dit le soit de façon certaine et vérifiée et avec pour fondement, vérité et autorité [il convient ainsi] car comme cette chose est très importante dans l'église chrétienne, tant pour la fermeté de la foi que pour la bonne instruction et l'exemple à donner aux fidèles [...] et en plus de cela, dans toute histoire, on exige surtout vérité et certitude, alors pourquoi ne pas en exiger autant pour l'histoire des saints pour lesquelles il est encore plus vrai qu'il importe de dire des choses certaines et véritables ${ }^{19}$.

Mais à ne vouloir retenir et représenter que les histoires véritables et donc recevables, il était possible d'éloigner le fidèle de ses propres modèles de dévotion. Ce fut la limite rencontrée par le concile de Trente, la pratique autorisant par la suite le maintien de certaines traditions. Il était devenu par exemple délicat de représenter l'histoire de la Vierge Marie, à commencer par celle du baiser d'Anne et de Joachim sous la Porte dorée, jusqu'à sa présentation au

\footnotetext{
${ }^{18}$ Ambrosio de MORALES, Lo que paresce se deve advertir para hazer..., op.cit. : «Por que en todo tiempo asi en esta matería como en todas las demas, huvo siempre escritores buenos y notables, y tanto mas pudieron y prevaleçieron en esta parte los no tales quanto la simplicidad poca advertida de algunos christianos blandos y piadosos, amó mas y precio aquellas cossas y las enseño y las encarecío a otros como cosas muy sanctas y llenas de devoción y bien muestra esto la santa yglesia rromana y mucho sentimiento ha hecho siempre dello pues muchos siglos atras le ha parecido que convenía remediar este daño y con gravisimos decretos tiene proveydo que escripturas de los hechos de los sanctos y que ystorias de los martires se ayan de escluir y desechar como apócriphas aunque sean muy antiguas en la yglessia [las cossas que la yglesia por sus decretos tiene y a proveydo que se excluyan y deshechen. Si en los licionarios de que al presente se usa ay algunas destas. Muy justo es que se quiten y pongan otras aprovadas en su lugar] y ayan venido de mano en mano en ella, - esto esta assy proveido muchos cientos años ha en la yglesia mas todavía se retiene y se conserva mucho desto, y se lee hartas vezes en ella añadiendose tanbien de nuevo otras cossas que pareçen mucho a las otras que primero havia por las piadossas caussas qua arríva decíamos. Todo esto se ha de dexar en las leçiones y buscar la çertidumbre en los autores antiguos en quien tanbien ha de haver mucho cuydado al escoger. »

${ }^{19} \mathrm{Ibid}:$ : [...] conviene mirar con gran cuidado y effectuarlo despues con mucha diligencia que todo lo que se dixere sean cosas çiertas y averiguadas y que tengan fundamento de mucha verdad y auctoridad [conviene assy] porque siendo una cossa esta en la yglesia christiana muy importante assi para la firmeça de la fee commo para la buena instituçíon y exemplo de los fieles [...] y fuera desto en qualquier ystoría se pyde prinçipalmente verdad y çertidumbre pues porque no la emos de pedir tambien en la ystoría de los sanctos donde es mucha rrazon y importa mucho mas que se digan cossas çiertas y verdaderas ».
} 
Temple. Cette tradition, d'origine apocryphe, n'en restait pas moins soutenue par la croyance commune, ou « vulgaire », comme la nomme le frère José de Sigüenza, historien de l'ordre de saint Jérôme et du Palais-monastère de l'Escorial, manifestement sensible au problème posé par le choix entre les injonctions tridentines et la tradition. Il écrit à propos des noces de la Vierge avec Joseph, que la version retenue pour le cloître bas du monastère de l'Escorial était celle admise le plus communément : «Dans toutes ces histoires, comme je l'ai dit, on retient ce que le vulgaire considère en la matière $»^{20}$. La présence de ces représentations de l'enfance de Marie s'explique, sans doute, par l'appréciation des termes conciliaires " fausse doctrine ", à propos desquels Molanus, l'un des premiers théoriciens de l'art religieux, apporte un avis éclairant en rapprochant ces termes de ceux qui les suivent: "erreur dangereuse ». La tradition apocryphe ou " fausse doctrine » ne doit être exposée si elle est porteuse d'une « erreur dangereuse » comme par exemple la Bienheureuse Vierge en couches, qui ne doit être montrée alitée et souffrante, ce qui est contraire à la doctrine catholique de l'enfantement sans douleur de Jésus. Au contraire, les «fausses doctrines » qu'il considère sans danger, comme la présentation de Marie au Temple ou Marie embrassant les bras du Christ, peuvent être gardées «mais non sans vigilance». Pour Molanus, sauf avis contraire d'un concile provincial, « il n'est pas utile de faire diparaitre ou de modifier ce genre de peintures $»^{21}$.

En Espagne, avant même la publication du traité de Molanus, il semble que Philippe II ait agi selon la même opinion, d'après ses annotations au mémoire d'Ambrosio de Morales, daté de 1566, et dans lequel l'historiographe du roi se montrait partagé entre la nécessité de faire table rase des récits apocryphes, "pour informer et enseigner aux chrétiens, il conviendra de les remplacer par des [récits] plus sûrs et vérifiés ", et celle de préserver les traditions dévotionnelles qui reposent sur ces récits, « la dévotion de certains reposant sur une humilité seule » en ne perdant pas de vue l'éducation et l'édification des chrétiens. Annotant le texte d'Ambrosio, Philippe II tranche par un compromis consistant à ne rien modifier de l'existant, donc conserver les traditions mêmes fausses, sauf si celles-ci peuvent être remplacées par des versions plus certaines, sinon «on ne devrait pas remplacer ce que l'Église a admis jusqu'à présent $\gg{ }^{22}$.

\section{De la dignité des saints}

[...] enfin toute indécence sera évitée, en sorte que les images ne soient ni peintes ni ornées d'une beauté provocante ${ }^{23}$.

Avec la contre-offensive catholique, l'art de la Renaissance finissant jeta un voile pudique sur son amour de la nature. Le concile de Trente, appelant à la réalisation d'images dignes, fut suivi de plusieurs commentaires nouveaux sur l'art de la peinture à commencer par le traité d'Andrea Gilli da Fabriano, Dialogo degli errori dei pittori, publié à Camerino en 1564. L'ouvrage discutait alors d'un sujet similaire au débat sur la pudicité des fresques de la chapelle sixtine, dont les nus avaient été en partie recouverts par Volterra sous Paul IV.

\footnotetext{
${ }^{20}$ Fray José de SIGÜENZA, Historia de la Orden de San Gerónymo, Tercera Parte, La fundación del Monasterio de San Lorenzo el Real: Fábrica del Rey don Felipe Segundo, Madrid, 1605, Biblioteca de Autores Españoles, XXX, p. 543 : «En todas estas historias, como adverti, se va presuponiendo lo que el vulgo tiene recibido en esto $»$.

${ }^{21}$ MOLANUS, op. cit., p. 204.

22 Ambrosio de MORALES, «Lo que paresce se deve advertir... », op.cit. : «para ynfomar y enseñar los chrístianos converna mucho poner en lugar destas otras cossas mas çíertas y averiguadas », «la devoción de algunos, asegurada con una simple umildad » et « no se avría de quitar lo que la yglesia aya admitido ».

${ }^{23}$ Décret cité en note 6.
} 
L'auteur, lui, s'en prenait à la valeur morale discutable des fresques du Vatican. L'ouvrage véritablement fondateur de la nouvelle iconographie de la réforme catholique fut celui de Molanus, Jean Ver Meulen, publié en 1570 sous le titre de De historia sacrarum imaginum et picturarum pro vero earum usu contra abusus. Né à Lille en 1533, Molanus fit ses études à l'université de Louvain où il obtint le grade de docteur en 1570. L'année suivante, Philippe II le désignait comme censeur des livres et il se montra un sujet loyal dans le contexte troublé des guerres des Pays-Bas. Quant à son ouvrage, il traite moins de l'art que de la théologie dont il est le support, renvoyant à l'existence réelle de ce que la représentation donne à voir en terme de signification et non de ressemblance. Fixant parfois de nouveaux modèles, le traité procède surtout par la condamnation de l'art religieux existant, dressant le vocabulaire iconographique des saints et de l'histoire sainte. L'ouvrage de Molanus demeura une autorité pour l'époque avec le Discorso intorno alle imagini sacre et profane de Paleotti, publié à Bologne en $1582^{24}$.

À travers ces traités, la dignité se révèle d'abord par la quasi-disparition du nu. La «beauté provocante» des belles anatomies de la Renaissance fut jugée impie et désormais «la décence devint le caractère essentiel de l'art religieux » ${ }^{25}$. Paleotti préconisait même l'examen de toute peinture religieuse par une commission ecclésiastique, ce que fit d'ailleurs l'Inquisition espagnole en nommant des inspecteurs de tableaux de piété qui traquèrent l'indécence dans les tableaux et sur des objets courants tels que tabatières, éventails et miroirs $^{26}$. Francisco Pacheco, censeur des peintures sacrées pour l'Inquisition et auteur d'un Traité de la peinture publié en 1649, y enseignait aux artistes à se conformer à l'autorité des Écritures. En 1730, lorsque Juan de Ayala publiait son Pictor christianus eruditus, les préceptes établis après le concile relevaient des mêmes enjeux. Molanus avait démontré, en son temps, la vertu d'enseignement et de puissance des images et qu'à ce titre les images indécentes devaient être bannies. Ayala soulignait aussi que la nudité des corps n'est pas autorisée dans les images saintes et jugeait néfastes les nus de l'Antiquité, opposant l'innocence à l'impudence et à la concupiscence. Il préconisait donc la plus grande décence pour des nus imposés comme ceux d'Adam, d'Eve et de certains anges. Quant aux martyrs et vierges, « omnino nudos effigiari [...] cum panis aut subligaculis circa partes illas » afin de préserver la dignité des chrétiens ${ }^{27}$. Du cinquième au huitième livre, tous consacrés à l'image des saints, l'auteur élabore tout un répertoire des idées et des formes à retenir pour les saints : une image du martyre de saint Laurent doit signifier la victoire contre Rome symbolisée par un centurion tandis que le tourment du saint figure les instruments du supplice. Quant à saint Jacques, fils de Zébédée, premier apôtre et patron de l'Espagne, qu'il soit représenté en pèlerin ${ }^{28}$ ou en martyr, occis par le glaive.

La répétition d'un canon pictural simple s'explique dans une perspective dévotionnelle, aucun détail superflu ne devant éloigner le sujet de l'objet de sa dévotion comme le préconisait le cardinal Paleotti en 1582, dans son Discorso. Les sujets peints étaient donc traités avec la plus grande sobriété tendant à faire disparaître les « figurants inutiles $»^{29}$,

\footnotetext{
${ }^{24}$ Cardinal Gabriele PALEOTTI, Discorso intorno alle imagini sacre et profane diviso in $V$ libri dove si scuoprono varii abusi loro, et si dichiara il vermo modo che christianamente si doveria osservare nel porle nelle chiese, nelle case et in ogni altro luogo, raccolto et posto insieme ad utile dell'anime per commissionne di Mons. Illustriss. Et Reverendis. Card. Palaeotti, vescovo di Bologna, Bologne, 1582.

${ }^{25}$ Emile MÂLE, L'art religieux de la fin XVIe siècle, du XVIIe siècle et du XVIIIe siècle. Étude sur l'iconographie après le concile de Trente, Paris, A. Colin, 1972, p. 3.

${ }^{26}$ Ch. DEJOB, De l'influence du concile de Trente, Paris, 1884, p. 258.

${ }^{27}$ Juan de AYALA, Pictor christianus eruditus, Madrid, Conventus, 1730, p. 12.

28 «Pingitur enim peregrini habitu, oblongo innixus baculo, ex quo etiam bursa pendeat, \& cira humeros amiculo, quod Hispani Esclavinam vocant; insuper \& cum galero satis amplo, quem tamen ornant conchae, que circa litus maris passim se osserunt », AYALA, op. cit., p. 320.

${ }^{29}$ Emile MÂLE, op.cit., p.6.
} 
comme un paysage, pour ne laisser que le sujet religieux et fixer l'attention du fidèle sur l'espace entièrement sacré de la représentation. Toute l'attention devait être portée sur la figure du saint d'où une conception de la dignité relevant aussi de la noblesse donnée aux personnages sacrés. Cette noblesse était révélée à travers l'efficacité discursive du martyre, saisissant, avec un sens certain du pathétique, le moment le plus édifiant de la vie du saint. L'image du martyre, évocatrice de la souffrance pour le simple mortel, procède en réalité par antiphrase, figurant non pas l'apparence du supplice mais la joie du martyr entrant dans la gloire de Dieu. L'image est alors doublement opérante, enseignant l'histoire du martyr et édifiant le chrétien dans l'exemple du saint. La vertu de puissance de l'image en rendait la prédication plus efficace. Pedro de Ribadeneyra, dans sa préface au Flos sanctorum, conseillait, pour mieux guider et inspirer les chrétiens, de respecter la règle de la brièveté pour ne pas rendre ennuyeux le récit trop long de la vie d'un saint et de n'en retenir que les faits les plus remarquables comme les miracles ou le martyre. Néanmoins, les peintures et liturgies des saints n'optèrent pas exclusivement pour le récit du martyre. Les œuvres de Navarrete, comme le martyre de saint Jacques en 1571, son bourreau lui tranchant la gorge, et celles plus nombreuses représentant le martyre de saint Laurent sur le gril, n'occupaient pas une place de choix dans l'édifice escurialense, où se trouvaient également des représentations de la vie du diacre au collège et dans le chœur ainsi que dans les ornements vestimentaires pour le jour de la saint Laurent. Le choix des ornements fut notifié dans des documents pour l'organisation des offices de l'église de l'Escorial, aujourd'hui conservés à Simancas et d'après lesquels, il apparaît que le martyre de saint Laurent devait figurer sur le frontal tandis que les autres faits notables des derniers moments de sa vie occupés par la mort de Sixte II, la distribution du trésor aux pauvres et ses miracles figuraient sur la chasuble, les dalmatiques et les capes du chœur $^{30}$. Si le martyre, en tant que vision de la foi, devenait l'occasion privilégiée d'un discours pictural sur la grâce, les images des vies des saints restaient encore le support d'un enseignement en insistant sur leur noblesse.

L'idée de noblesse pouvait inspirer différemment les peintres au risque de déplaire aux commanditaires, comme ce fut le cas pour le Caravage, dont la noblesse des apôtres consistait en leur pauvreté. La stricte humilité de son saint Matthieu en guenille fut rejetée, jugée vulgaire et indécente. La noblesse pouvait aussi être marquée par le respect de la vraisemblance. Paleotti conseillait de ne pas utiliser de modèles vivants pour les figures saintes, encore moins des personnes de mauvaise vie ${ }^{31}$. Cette précaution avait aussi été donnée par Molanus pour éviter toute vanité, peu en accord avec la sainteté du sujet ${ }^{32}$. Fut-ce au nom de l'humilité de l'œuvre sacrée ou par souci de préserver sa propre grandeur que Philippe II relégua, dans une autre chapelle, le Saint Maurice et la légion thébaine du Greco, réalisé en 1579, dont l'un des compagnons du saint avait, comme le pense John Bury, les traits d'Emmanuel-Philibert de Savoie et saint Maurice, ceux d'Alexandre Farnèse ${ }^{33}$ ? Le refus de cette œuvre relève de la trop grande place accordée à la discussion de saint Maurice rapportant le message de l'empereur, au premier plan, tandis que le martyre se déroule en second plan. Le refus relevait sans doute aussi du souci de Philippe II de n'exposer le portrait d'aucun membre de sa famille, inférieur en dignité, comme dans sa galerie de portraits du Pardo. En revanche, la représentation de sainte Anne, réalisée en 1584 par Luca Cambiaso et figurant, selon Sigüenza, la reine Anne d'Autriche, demeura, sans soulever l'indignation, dans la première chapelle latérale de l'église, du côté de l'Évangile.

\footnotetext{
${ }^{30}$ Memoria de los ornamentos Reales que se han de hazer..., AGS, Casas y Sitios Reales, dossier 260, fol. 318.

${ }^{31}$ Ch. DEJOB, op. cit., p. 248.

${ }^{32}$ MOLANUS, op. cit., p. 228.

${ }^{33}$ John BURY, «El « Martirio de San Mauricio y la Legión tebana » de El Greco » dans Reales Sitios, XXIV, n91, 1987, pp. 20-36.
} 
La question du portrait de cour ou d'État renvoie au principe de la vraisemblance par la ressemblance qui présidait alors à leur réalisation tandis que dans le domaine de l'art religieux, la dignité des saints passait par un souci de vraisemblance, dans le sens du probable. Le non-respect de cette règle a pu conduire certaines œuvres à être rejetées comme celle de Federico Zuccari pour le compte de Philippe II. Précédé par sa renommée, le peintre et théoricien de l'art Federico Zuccari s'était rendu à Madrid en 1585, sur invitation de Philippe $\mathrm{II}^{34}$. Il s'était vu confier la tâche de réaliser des œuvres pour le Belvédère et le palais Farnèse. Il fut chargé (grâce à la disparition de Navarette) de peindre le grand retable de l'Escorial. Il réalisa ainsi pour les côtés de la custode, une Nativité de l'enfant Jésus et l'Adoration des Mages. Quand il les acheva, Zuccari « quedó tan enamorado de sus manos », qu'il voulut faire voir au roi ces deux œuvres, avant de les installer. Confiant, le peintre les plaça sous la meilleure lumière et déclara au roi, d'après Sigüenza, qu'elles montraient quel pouvait être l'aboutissement de l'art car, il convenait de les voir autant de près que de loin. Le roi lui rendit grâce et fit bonne figure sans dire un mot, se contentant d'observer quand son attention s'arrêta sur un détail incongru de l'Adoration de Mages. Il s'agissait d'un panier rempli d'œufs qu'offrait à la Vierge l'un des bergers venus adorer l'enfant Jésus :

[...] cela semble impropre qu'un pasteur quittant son bétail à minuit, et encore en courant, puisse être venu avec tant d'œufs s'il ne possède pas de poules ${ }^{35}$.

Par ce détail, l'œuvre n'était plus vraisemblable et donc devenait impropre à la dévotion. Federico Zuccari était pourtant expert en sujets religieux et lorsqu'il avait réalisé des œuvres pour le palais du cardinal Farnèse à Caprarola, toutes les indications nécessaires concernant vêtements, objets et couleurs lui avaient été données comme tant d'autres artistes sollicitaient l'avis de théologiens. Zuccari savait respecter les règles de cet art religieux devenu exigeant, et pourtant ces œuvres ne surent plaire, malgré l'estime de Philippe II pour l'artiste ${ }^{36}$ :

De tout ce qu'il a pu peindre, peu ont contenté le roi et quiconque, et rien de ce qu'il fit ne fut à la hauteur des espérances que son nom avait suscitées ${ }^{37}$.

Arrivé avec tous les honneurs dus à sa réputation, Frederico Zuccari fut remercié cinq ans plus tard avec toutes les faveurs qu'un grand prince sait accorder pour son service. Zuccari reçut par contrat six mille ducats, ses services ayant été estimés à deux mille ducats par an avec huit mille et quatre cents ducats pour sa venue et ses frais de départ. Dès que l'artiste fut reparti pour l'Italie, Philippe II fit déposer ses œuvres du retable, dont le fameux Martyre de saint Laurent, les reléguant dans d'autres chapelles et salles du couvent. Philippe II fut un mécène difficile à satisfaire car, de l'avis même de Sigüenza qui eut peu l'occasion de voir les œuvres de Zuccari, celles-ci « sin duda son de lo mejor que aquí nos dexó ».

D'après Sigüenza, la disparition de Navarrete le Muet en 1579, avait donné à l'Espagne la chance d'acceuillir de grands peintres italiens, Federico Zuccari et Luca Cambiaso. Leur réputation ne fut pas à la hauteur des attentes de Philippe II qui renonça à plusieurs de leurs œuvres pour l'église: les fresques de Zuccari pour le cloître bas, représentant la vie de la Vierge, avaient été réalisées par ses apprentis et déplurent fortement

\footnotetext{
${ }^{34}$ José de Sigüenza se fait l'écho de cette réputation : «Vino Frederico con tanto nombre endereçado al servisio del Rey, por medio de personas tan graves y de tan buen juyzio, y las estampas suyas le avian hecho tan famoso, que poco menos le salieramos a recebir con palio », op. cit., p. 563.

35 José de SIGÜENZA, op. cit., p. 563: «[...] que parece impropria un pastor que venia de su ganado a media noche, y aun corriendo, pudiesse aver allegado tantos huevos, si no guardava gallinas. »

${ }^{36}$ Fernando CHECA, Felipe II. Mecenas de las artes, Madrid, Nerea, 1992, p. 325.

${ }^{37}$ José de SIGÜENZA, op.cit., p. 563 : «Todo esto pintó y poco dello dió contento al Rey ni a nadie, y ninguna cosa hizo que llegasse con mucho a las esperanças que se avian concebido de su nombre. »
} 
au roi qui les confia à Tibaldi ; les Onze mille vierges et le Saint Michel de Luca Cambiaso (1584), destinés aux chapelles de l'église, se retrouvèrent finalement au collège. Le tableau de Saint Michel ne répondait pas aux règles énoncées plus haut, faisant du tableau une œuvre peu convaincante : l'ange, d'assez bonne figure, semblait disparaître au milieu de démons « fieros, desnudos, en posturas estrañas y para altar feas, poco pías ${ }^{38}$. L'œuvre péchait par absence de dignité, comme celle des Onze mille vierges paraissait peu vraisemblable :

\begin{abstract}
Dans celui des Vierges, bien qu'il en ait disposé quelques-unes, elles étaient peu nombreuses par rapport au nombre qu'elles furent, ce qui ôte l'envie de les prier, et il y a un seul bourreau qui est en train de les décapiter (il savait s'y prendre), qui bien qu'ayant le visage gracieux, est laid, mal habillé, et le coloris en général paraît sans couleurs et délavé, et malgré toutes ces fautes, on ne peut le nier, on découvre néanmoins toute la vaillance du maître, sa grande connaissance et son habileté à établir les figures, et montrer sans difficulté toutes les parties avec une juste proportion et mouvement ${ }^{39}$.
\end{abstract}

Sigüenza sait reconnaître le talent de ces deux artistes italiens comme il sait reconnaître une œuvre religieuse conforme aux règles de la dignité et de la vraisemblance.

Les critiques que formule Sigüenza portent souvent sur l'excessive pâleur des couleurs, "un colorido muerto », sur le peu d'ornement des figures manquant ainsi de noblesse. Un principe semble donc présider au succès des images religieuses, celui de la beauté. Les théoriciens de l'art s'opposent, à la même époque, sur leur conception du beau, héritée alors de l'Idée platonicienne. L'idée de la beauté se définissait sur le mode du divin qui inspire la création de l'artiste pour toucher les sens et solliciter l'intellect. Lorsqu'à l'Escorial, artistes espagnols et italiens s'attèlent à sa décoration, la théorie de l'art fait défaut en Espagne. Ce fut pour combler cette lacune que Pacheco, prenant appui sur le modèle italien des traités d'art et sur la référence aux auteurs antiques et des Pères de l'Église, établit une doctrine du beau, reposant, pour une grande part, sur une imitation de la nature limitée par la décence. Pragmatique, Pacheco recherche dans la nature, la vraisemblance de l'Idée. Celle-ci ne prendrait corps et donc du relief qu'à travers une œuvre parfaite de lignes et de couleurs imitant au mieux la nature et ses proportions. Comme pour Sigüenza, précieux observateur de l'art escurialense, la couleur est la touche finale à l'harmonie des formes, seules capables de donner de la beauté à l'œuvre picturale. La beauté que recherche Sigüenza dans les peintures des saints se rapproche de la théorie platonicienne de l'harmonie des formes que l'artiste retrouve par l'imitation des proportions et des mesures. Cette nature déterminée par l'ordre et l'harmonie, révélant la beauté de l'œuvre divine, fut préétablie dans le Timée de Platon. Dans les peintures des saints, l'artiste imite à la fois ce qui est visible, un modèle extérieur défini par des canons iconographiques, et ce qui est imaginé par l'entendement dans le respect de la doctrine catholique. La vraisemblance se fait non seulement imitation de la nature mais aussi, par conséquent, expression d'une immanence divine. L'Idée, assimilée à la beauté, devient une manifestation visible du bien qui a son origine en Dieu. Retranscrite par l'art pictural, cette idée s'exprimait par ce que Sigüenza nomme le «decoro », principe dominant l'art religieux marqué par l'austérité. Le « decoro » consistait en la suppression de la nudité mais aussi et surtout dans le respect de la pure doctrine. Ainsi Sigüenza jugea en effet indécent le martyre de saint Pierre du Titien dont il considérait l'intention fuyante du visage indigne de l'idée de grandeur de l'apôtre martyr.

\footnotetext{
${ }^{38}$ Ibid., p. 562.

${ }^{39}$ Ibid.: «En el de las Virgines, aunque puso algunas, para el numero que pudiera significar fueron muy pocas, y aquellas de suerte que quitan la gana de rezar en ellas, y un solo verdugo que las esta descabeçando (tenía bien en que entender), que aunque la figura es ayrosa, es fea, mal vestida, y el colorido de todo ello descolorido y deslavado, y con todas estas faltas, no se le puede negar sino que descubren la valentia del maestro, lo mucho que sabia y quan diestro era en plantar las figuras, y mostrar sin dificultad todas las partes, con singular proporción y movimiento. »
} 


\title{
La simplicité du lexique pour montrer le chemin
}

L'une des vocations, sinon la principale, de la représentation des saints était pédagogique afin de montrer le chemin de la foi par l'exemplum, c'est-à-dire le rôle d'imitation du martyr, qui accomplit l'extraordinaire par sa mort, ou encore le saint, qui se distingue aussi par sa capacité à devenir extraordinaire. La contemplation des martyrs et des saints se fait parole visuelle et devient une forme d'oraison méditative voire célébrante pour obtenir l'intercession du saint, pratique défendue de toute accusation d'idolâtrie par le concile de Trente lors de sa XXVe session :

Aussi leur enseigneront-ils [les évêques] que les saints qui règnent avec le Christ offrent à Dieu leurs prières pour les hommes ; qu'il est bon et utile de les invoquer humblement [...], de recourir à leurs prières, à leur aide et à leur assistance ${ }^{40}$.

Dans la perspective de l'imitation d'un modèle, l'hagiographie picturale avait recours à une grammaire commune avec les sermons. Peintures et sermons devaient aussi partager une préoccupation commune, celle de perfectionner l'éloquence de la prédication contre la corruption de la parole évangélique par des textes apocryphes. De même que les sermons se construisent sur une parole d'évangile véritable, la représentation d'un saint en exprime tout le sens. Le martyre de saint Jacques, par exemple, représenté par Navarrete le Muet, insiste sur le don de soi comme démonstration de la foi. Premier apôtre à mourir pour sa foi par le glaive, saint Jacques devient un discours du don dont le sens est résumé par cette parole de Matthieu $(20,28)$ : «C'est ainsi que le Fils de l'homme n'est pas venu pour être servi, mais pour servir et donner sa vie en rançon pour une multitude » ou encore (Matthieu 20, 23) « Vous boirez ma coupe » à l'intention des fils de Zébédée. L'image du martyre remplit une double fonction d'imitation; imitation par le fidèle et imitation de la mort du Christ. L'art transpose ainsi ce que la réforme attend des images, non pas une vaine croyance en leur capacité propitiatoire, mais la transposition du modèle christique :

\begin{abstract}
Aussi, à travers les images que nous baisons, devant lesquelles nous nous découvrons et nous prosternons, c'est le Christ que nous adorons et les saints, dont elles portent la ressemblance, que nous vénérons. C'est ce qui a été défini par les décrets des conciles, spécialement du deuxième concile de Nicée contre les adversaires des images ${ }^{41}$.
\end{abstract}

De là, également, une intériorisation aisée de la figure du martyr par sa dimension humaine. Le sermon prononcé par Francisco Fernández Galvan ${ }^{42}$, consacré à l'apôtre Jacques, commençait, de cette façon, par souligner la ressemblance entre ses faiblesses et celles de la

\footnotetext{
${ }^{40}$ Décret cité en note 6.

${ }^{41}$ Idem.

${ }^{42}$ Francisco FERNÁNDEZ GALVAN, Sermones de las festividades de los santos. Traduzidos de la lengua portuguesa en la Castellana, por don Antonio de Azevedo, y San Portugues, Madrid, Viuda de Alonso Martin, Madrid, 1615, fol. 206v [première édition portugaise et posthume en 1611 à Lisbonne chez Pedro Craesbeeck]. D'après la Bibliotheca lusitana de Machado (tome II, Lisboa, 1747, p. 114), Francisco Fernández Galvan ou Galvam (1554-1610) était originaire de Lisbonne. Il fit partie des domestiques de la Maison des infants Isabela et Duarte puis étudia la Rhétorique et la Philosophie au collège d'Evora avant d'obtenir le grade de Maître ès Arts de l'université de Coïmbre et enfin celui de docteur en théologie. Il exerça, avec succès, son ministère de prédicateur à la cour de Lisbonne pendant une douzaine d'années avant de partir pour Rome en 1585 où il occupa plusieurs charges ecclésiastiques. De retour dans la Péninsule, en 1590, il prêcha dans la chapelle royale de Madrid et dans le couvent royal des Descalzas Reales sans doute jusqu'à sa mort à l'âge de 56 ans.
} 
multitude qui sera sauvée par la puissance de la grâce divine. Les imperfections de saint Jacques sont celles de tous et par là plus édifiantes pour agrandir la gloire et le pouvoir de Dieu, lequel «sait faire de ces gens faibles, les fermes colonnes de son église " ${ }^{43}$. Comme saint Jacques, laisser les honneurs pour les souffrances du martyre. Les sermons pour la fête de l'apôtre comportaient souvent une partie basée sur l'évangile tandis qu'une seconde était plus circonstanciée, soulignant le rapport entre le saint et l'Espagne depuis son rôle présumé d'évangélisateur et de défenseur armé, en résumé de saint patron de l'Espagne ${ }^{44}$. Dans le tableau de Navarrete, c'est comme si le peintre avait peint ce qu'il avait pu entendre d'un sermon prononcé en l'honneur de saint Jacques : la grandeur rédemptrice du martyre et, en arrière-plan, le saint combattant les Maures.

Les saints les plus fréquemment représentés à l'Escorial ont chacun une parole $\mathrm{du}$ Christ à transmettre pour trouver le chemin d'oraison : saint Jacques et la grâce du martyre, saint Laurent et le triomphe par le martyre, ou encore saint Jérôme et la pénitence. Ce dernier occupait une place privilégiée dans le programme iconographique des saints, Philippe II ayant choisi de doter de son monastère de l'Escorial, les hiéronymites, dont lui-même et l'empereur affectionnaient tout particulièrement la dimension contemplative et orante ${ }^{45}$. L'expérience spirituelle de saint Jérôme en Orient est le moment exemplaire servant la dimension contemplative de l'ordre et se trouve donc à l'origine d'un canon iconographique : le saint en pénitence, vivant en ermite avec la seule compagnie d'un lion. La nature, souvent luxuriante dans les représentations du saint pénitent, n'avait rien à voir avec le désert de Chalcis, en réalité symbolisé par le lion. Navarrete le Muet, comme le Titien, avait opté pour le choix insolite d'une nature verdoyante, ce que ne manqua pas de lui reprocher José de Sigüenza. Définissant l'œuvre, livrée en 1571 à l'Escorial, comme étant saint Jérôme en pénitence et au désert, Sigüenza commence par s'intéresser au saint, à genoux et à moitié nu, se frappant la poitrine avec une pierre. Sigüenza trouve la posture convaincante car « difícil », appréciant ainsi ce qui peut évoquer la souffrance. Le saint plaît, tant il traduit l'idée d'une vénérable vieillesse, parce qu'il est « beau, grave et plein d'un esprit véritablement saint ». Le lion, bien visible près d'une source, est une "belle bête » qui semble également convaincre le moine mais il en est tout autrement du paysage alentour, "plein de fraîcheur et arboré ». D'après Sigüenza, ce paysage ne convient pas car il ne correspond pas au choix de saint Jérôme pour sa pénitence, qui nécessite davantage «comme il le dit, un dur et âpre désert » ${ }^{46}$. Et pourtant, en 1568, les moines hiéronymites offrirent à Philippe II un bréviaire enluminé dans lequel figure saint Jérôme pénitent au milieu d'un désert verdoyant ${ }^{47}$. Sigüenza n'a sans doute pas saisi alors ce que peintres et sermons comprirent de la pénitence de saint Jérôme. Individu dans le monde, le saint prie pour le réformer, mais il est tout autant hors-le-monde par sa solitude pénitente, laissant peu d'importance à ce qui l'entoure. Si la vision douce d'un paysage verdoyant l'emporte sur celle de l'âpre désert, c'est que le chemin difficile de la vertu n'est que «dulçura » pour le saint. La représentation doit pouvoir signifier à la fois les

\footnotetext{
${ }^{43}$ Francisco FERNÁNDEZ GALVAN, «Sermon en la fiesta del Apostol Santiago, Lisboa en la yglesia de Santiago. Año 1606 », op.cit., fol. 178v: « de gente tan flaca sabe hazer la colunas firmes de su yglesia ».

${ }^{44}$ Voir deux exemples très similaires de sermons en l'honneur de saint Jacques dans Francisco FERNÁNDEZ GALVAN, «Sermon en la fiesta del Apostol Santiago, Lisboa en la yglesia de Santiago. Año 1606 », op.cit., fol. 177r.-184v., et Andrés PÉREZ, «En la fiesta del glorioso apostol Santiago, patron de España », dans Tomo segundo de los sermones de los santos, Valladolid, 1622, pp. 59-88.

${ }^{45}$ G. SABAU BERGAMÍN, « Relaciones de Felipe II con la Orden de San Jerónimo », in Studia Hieronymiana, 2, 1973, pp. 313-346, et G. SÁNCHEZ MECO, El Escorial y la orden jerónima : análisis económico-social de una comunidad religiosa, Madrid, Patrimonio Nacional, 1985.

${ }^{46}$ SIGÜENZA, op.cit., p.549 : « hermoso, grave y lleno de espiritu verdaderamente de santo »; « linda bestia »; « de mucha frescura y arboleda »; « como el dize, un desierto fiero, aspero ».

${ }^{47}$ Francisco M. GIMENO BLAY, Henry KAMEN, «Les Heures du roi. Le Bréviaire de Philippe II », in FMR, n72, Paris, février-mars 1998, pp. 65-76.
} 
conditions visibles, apparentes de la souffrance et l'idée que pour un saint et un martyr, elles sont au contraire douces en leur ouvrant la voie vers Dieu ${ }^{48}$. Sigüenza considérait sans doute que la représentation artistique n'avait pas le pouvoir de transcender les apparences alors qu'il en saisissait parfaitement la subjectivité dans les discours d'une autre teneur, sensible par sa formation à ce que les sermons pouvaient donner comme explication concernant la souffrance des saints, à savoir que : « les pierres paraissent des émeraudes à saint Étienne, les grils, des roses à saint Laurent, le désert, un paradis à saint Jérôme ${ }^{49}$. Le désert n'apporte pas la souffrance au pénitent mais il est un paradis dans lequel il gagne sa sainteté, une idée exprimée par les sermons comme par les représentations artistiques. Ce que Sigüenza n'avait pas saisi, c'est une mystique se réalisant dans un ravissement extatique et que l'art annonça précocement avec la visualisation baroque de la piété.

L'idée de sainteté doit être suffisamment explicite pour faire de la représentation une image de dévotion. Sigüenza associe, à ce propos, la dévotion et la beauté au sens de l'esthétique de l'harmonie des formes et des couleurs mais également, et surtout, dans la manière de figurer l'Idée transmise par tel personnage biblique ou tel saint ultérieur. En effet, la représentation hagiographique est une narration difficile en ce qu'elle doit relever plusieurs défis, dont le principal est d'accorder l'esthétique picturale avec les impératifs tridentins du discours théologique. L'image sainte doit pouvoir induire une dévotion sans abus évoquée plus haut :

\begin{abstract}
Les évêques enseigneront avec soin que, par le moyen de l'histoire des mystères de notre rédemption représentés par des peintures ou par d'autres moyens semblables, le peuple est instruit et affermi dans les articles de la foi, qu'il doit se rappeler et vénérer assidûment. Et l'on retire aussi grand fruit de toutes les images saintes, non seulement parce que sont enseignés au peuple les bienfaits et les dons que lui confère le Christ, mais parce que, aussi, sont mis sous les yeux des fidèles les miracles de Dieu accomplis par les saints et les exemples salutaires donnés par ceux-ci : de la sorte, ils en rendent grâces à Dieu, ils conforment leur vie et leurs mœurs à l'imitation des saints et sont poussés à adorer et aimer Dieu et à cultiver la piété ${ }^{50}$.
\end{abstract}

La difficulté à représenter une scène hagiographique ou celle d'un évangile est d'autant plus appréciée qu'elle est réussie, comme Sigüenza le souligne par des superlatifs lorsque luimême est convaincu devant telle «hermosissima pintura » (le saint Jacques de Navarrete), « devocion y hermosura » (le Christ en croix de Tibaldi), " de mucha devocion » (le saint Michel de Tibaldi) ou encore «le premier en dévotion, majesté, piété, révérence qu'est le Christ à la colonne [...] Le visage plein de tristesse, de beauté et de gravité $"{ }^{51}$ (Navarrete). La dévotion est, en grande partie, déterminée par les choix narratifs et donc par la concordonce entre ce qui est su (entendu lors des sermons et plus rarement $1 u^{52}$ ) et ce qui est donné à voir. Cela aboutit à la mise en place de canons iconographiques propres à susciter la dévotion : saint Jérôme, "parce que sa pénitence [...] et sa doctrine éclaire[nt] l'entendement

\footnotetext{
${ }^{48}$ Dans l'édition française de son Flos sanctorum [Fleurs des vies des saints..., à Lyon chez Benoît Rigaud en 1625], le père jésuite Pedro de RIBADENEYRA souligne, à propos des tourments des martyrs, que les bourreaux « estoient plus las de frapper que les martyrs de souffrir, pour le grand désir qu'ils avaient d'endurer pour JesusChrist, à cause aussi du reconfort et de la joie que nostre Seigneur leur donnoit lors ».

${ }^{49}$ Francisco FERNÁNDEZ GALVAN, op.cit., fol. 279r. : « a S. Estevan le parecian las piedras esmeraldas, y a S. Lorenço las parillas rosas, a S. Geronímo desierto parayso ».

${ }^{50}$ Décret cité en note 6.

${ }^{51}$ SIGÜENZA, op.cit., p. 550 : « el primero en devocion, magestad, piedad, reverencia, es un Christo a la coluna $[\ldots]$ El rostro llena de tristeza, hermosura y gravedad ».

${ }^{52}$ Les sermons de Francisco Fernández Galvan étaient vendus 4 maravédis le pliego. Le prix de son recueil, publié en 1615 chez la veuve d'Alonso Martin, avait été fixé à 452 maravédis ou près de 13 réaux. D'après Bartolomé BENNASSAR (Valladolid au Siècle d'or, Paris, Mouton, 1967, p.277), le prix de la charge de blé, en période faste, pouvait baisser jusqu'à 30 réaux et l'azumbre de vin (2,016 litres) à 7 réaux en moyenne !
} 
humain $»^{53}$, est représenté en ermite pénitent ou encore saint Laurent, dont le martyre cruel révèle, au-delà des tourments et de la mort toute héroïque, le mystère de la Rédemption ${ }^{54}$, saint Jacques en pèlerin ou subissant le martyre, saint Philippe en évangélisateur ou crucifié ${ }^{55}$, etc.

Citant le discours sur les images sacrées du cardinal Paleotti, Francisco Pacheco énonce dans les années 1630, la capacité dévotionnelle des peintures sacrées et leur faculté à élever l'âme à Dieu :

\begin{abstract}
La peinture qui n'avait d'autre but que de ressembler à ce qu'elle imitait, acquiert maintenant, en tant qu'acte de vertu, un nouvel et riche habit en plus de la ressemblance, elle s'élève vers une fin suprême regardant vers la gloire éternelle, et cherchant à éloigner les hommes du vice, elle les guide vers le vrai culte de Dieu, Notre Seigneur. Les images chrétiennes ne se contentent pas de regarder vers Dieu mais encore vers le prochain ... car il n'y a pas de doute : toutes les œuvres vertueuses peuvent servir ensemble à la gloire de Dieu, à notre enseignement et à l'édification du prochain ${ }^{56}$.
\end{abstract}

Les auteurs de traités sur l'art de la réforme catholique n'ignoraient pas la difficulté à peindre les histoires sacrées. De leur exactitude dépendait la qualité de la dévotion. Il était donc nécessaire d'instruire et de conseiller les peintres pour la réalisation d'images de religion. Pacheco, relevant que le but essentiel des images est d'élever les âmes à Dieu, empruntait encore à Paleotti ses trois manières de vénérer les images sacrées :

[...] et bien que j'aie pu paraître m'éloigner quelque peu de mon discours en traitant de la matière des images, je tiens à dire que si ce n'est pas là tout l'emploi de la peinture, elles en sont cependant la partie la plus illustre et la plus digne de sa majesté, celle qui lui donne gloire et splendeur en illustrant les histoires sacrées, les mystères divins enseignés par la foi, les œuvres du Christ et de sa sainte Mère, les vies et morts des Saints Martyrs... C'est là la partie la plus difficile de cet art noble, à cause de ce que la vérité implique de nécessaire obligation, d'exactitude et de décence, ce que réussissent bien très peu d'artistes, même s'ils sont de grands peintres ${ }^{57} \ldots$

La répétition du même canon iconographique favorisait la mémorisation du modèle hagiographique. Ambrosio de Morales avait même songé à un programme d'inscriptions sacrées au-dessus des portes de l'édifice escurialense :

\footnotetext{
${ }^{53}$ Francisco FERNÁNDEZ GALVAN, op.cit., fol. 278r.: «porque su penitencia [...] y su dotrina alumbró los entendimientos humanos ».

54 À Rome, au début du IIIe siècle, l'archidiacre Laurent, consolé par l'annonce de sa mort prochaine pour l'honneur de Dieu, distribue les trésors de l'Église aux pauvres pour mieux « acquerir l'inestimable thresor de la couronne du martyre qu'il souhaitoit si ardemment ». À propos de saint Laurent, emprisonné puis supplicié, saint Augustin écrit dans les sermons aux saints : «Laurens estoit si fervent du desir de Jesus-Christ, qu'il ne sentoit point les traits de son persecuteur ; car tant plus l'ardeur de la foy est vive, de tant plus la flamme du supplice est amortie. Le feu matériel brusloit le corps du bien-heureux Laurens : mais l'amour intérieur dont son cœur estoit enflammé, adoucissoit l'ardeur extérieure. »(P. de RIBADENEYRA, Les Fleurs des vies saincts, Lyon, B. Rigaud, 1625, p. 627).

55 Saint Philippe l'apôtre, évangélisant la province de Phrygie, fut crucifié par les prêtres de Hieropolis : « [...] ils se saisirent du S. Apostre, et le menerent en prison, où apres l'avoir cruellement foüetté et le crucifierent et assommerent à coups de pierres, tandis qu'il remercioit nostre Seigneur de ce qu'il le faisoit imitateur de sa Croix » (P. de RIBADENEYRA, Les Fleurs des vies des saincts, Lyon, B. Rigaud, 1625, p. 372). L'apôtre fut sauvé par l'intervention divine qui engloutit les bourreaux et éntraîna la population à devenir chrétienne.

${ }^{56}$ Francisco PACHECO, L'Art de la peinture, Paris, Klincksieck, 1986, p. 46 : il cite le Discorso intorno alle immagini sacre du cardinal PALEOTTI, Bologne, 1582. Pacheco (1564-1644), peintre et érudit dirigeant l'académie de Séville, devint en 1618 censeur et visiteur des peintures sacrées du Tribunal de l'Inquisition de Séville. Il entreprit l'écriture de l'Art de la peinture dès les années 1630 mais l'ouvrage ne fut publié qu'en 1649. ${ }^{57}$ Idem.
} 
Il semble qu'il serait bon que toutes les pièces et officines ordinaires du monastère royal de Saint-Laurent de la Victoire, comme la loge, la sacristie, le chœur, le chapitre, la bibliothèque, etc. aient au-dessus de leurs portes des passages choisis de l'Écriture sacrée, de saints docteurs, de la règle ou d'autres bien méditées, qui puissent montrer, prévenir et admonester ceux qui entrent et passent par là. ${ }^{58}$

Le véritable profit, à faire parler les murs, n'était pas celui de l'ornementation. Les inscriptions sont tel un livre ouvert en permanence pour enseigner avec d'autant plus d'efficacité que les discours «par leur grande brièveté, sont facilement mémorisés » ${ }^{59}$. Ambrosio de Morales n'empruntait-il pas à Érasme quelques méthodes énoncées dans le De ratione studii composé pour Pierre Vitré ? L'humaniste rotterdamois préconisait, pour l'instruction des enfants, un travail de mémorisation reposant sur la lecture mais ne contestant pas que «la mémoire puisse être aidée par des lieux et par des images ». Il proposait d'aider la mémorisation des connaissances difficiles par leur exposition « aux murs de votre chambre afin qu'elles frappent votre vue à tout moment et même quand vous vous occupez d'autre chose », de même que les «formules brèves mais remarquables [...] seront [entre autres] peintes au-dessus des portes, sur les murs ou même sur les fenêtres vitrées, afin qu'il n'y ait pas de lieu où ne se présente à la vue quelque signe qui puisse aider la science ». La lecture répétitive passant par la compréhension, l'ordre et l'applicatoin servait à la connaissance des sciences pour Érasme tandis qu'Ambrosio restait dans un registre religieux mêlant l'image à la sentence édifiante. À la manière des peintures des saints, les inscriptions se mémorisaient par la répétition de la lecture, identique à celle du regard sur l'image. Les inscriptions devaient permettre d'élever les âmes des moines vers Dieu par la lecture et la réflexion collectives favorisant la discussion comme moyen de mémorisation. Ambrosio de Morales tenait d'autant plus à la réalisation de ce programme qu'il en faisait l'expérience quotidienne dans sa maison où les murs portaient des inscriptions pour éduquer les enfants amenés à les déchiffrer :

Qu'ensuite, les enfants, les découvrant [les murs de sa maison], désirent savoir ce que c'est : ce que cela signifie, et qu'ils le demandent aux autres, et l'ayant compris, qu'ils le gardent en mémoire, et les citent très naturellement. ${ }^{60}$

Il en allait de même dans le collège de l'Escorial où les murs des salles de cours enseignaient la Théologie, la Dialectique et la Physique à travers la peinture qui, loin de détourner les élèves de leur enseignement, « plus encore favorisera la mémoire, et le maître fera lecture de ce que les murs montrent aux yeux avec les couleurs ${ }^{61}$. La parole ou le regard, répétés et mémorisés, reposaient sur la simplicité et la brièveté du discours écrit ou iconique. De cette manière, chaque saint avait ses attributs et une histoire représentative. Le gril de saint Laurent suffit à définir le saint et son martyre tandis qu'une tradition plus ancienne choisissait indifféremment plusieurs moments déterminants de l'histoire du saint. Ce recours à un choix multiple d'événements ne favorisait guère une mémorisation efficace de l'exemplarité du

\footnotetext{
${ }^{58}$ Ambrosio de MORALES, Los titulos para las pieças del monasterio, [1566], AGS, Casas y Sitios Reales, dossier 258, fol. 243 : «Pareçe seria muy bien, que todas las pieças y officinas ordinarias del real monasterio de San Lorenço de la victoria, como porteria, sacristia, choro, capitulo, libreria etc tuviesen ençíma de las puertas algun buen dicho de sagrada escritura, de doctores santos, de la regla o de algunas cosas agudamente pensadas, con que hablasen, adviertiessen y amonestassen a los que entran y passan por alli ».

${ }^{59}$ Ibid. : « por su mucha brevedad se retienen façilmente en la memoria ».

${ }^{60}$ Ibid. : «Que luego los niños en viniendo a ellas [les murs de sa maison], dessean saber que son : y que significan, y lo preguntan a los otros, y quando lo saben, lo guardan en la memoria, y lo traen en la boca muy ordinarío ».

${ }^{61}$ Ibid. : «mas antes ayudara a la memoria, y el maestro leyera lo mismo que las paredes mostraran con los colores a los ojos ».
} 
saint. Le choix d'une histoire unique, résumant le moment capital de la sainteté du personnage, relevait d'un principe de simplicité capable de susciter la dévotion.

L'image hagiographique faisait ainsi œuvre de pédagogie par l'exemple en des termes là encore définis au concile de Trente :

Ils leur enseigneront ce que tous doivent savoir pour leur salut et leur apprendront brièvement, et en un langage facile, les vices dont il faut s'écarter et les vertus qu'il leur faut pratiquer pour qu'ils puissent échapper aux peines éventuelles et obtenir la gloire céleste $^{62}$

La simplicité du discours était une vertu rhétorique appréciée de la culture humaniste qui l'avait empruntée au traité oratoire de Quintilien. La réforme de l'éloquence de la chaire emprunta ainsi aux antiques l'art de la parole ${ }^{63}$. Louis de Grenade, auteur d'un grand nombre de sermons, rédigea à la fin de sa vie un commentaire à l'usage des prédicateurs pour apprendre l'éloquence de la chaire. Dans La Rhétorique de l'Eglise ou l'éloquence des prédicateurs, il reconnaît emprunter beaucoup aux maîtres de l'éloquence et de la rhétorique, et en particulier à Cicéron dont il reproduit l'art de l'éloquence. La vertu, s'il en est, de la simplicité narrative permet une meilleure lisibilité, une compréhension plus immédiate du contenu $^{64}$. L'enjeu des images était celui de l'éducation des fidèles en leur procurant un enseignement de la sainteté reposant sur une connaissance règlementée des récits hagiographiques grâce à l'exposé pictural. Ce fut en application du décret tridentin sur le catéchisme, missels et bréviaires du 3 décembre 1563, qu'Ambrosio de Morales avait proposé un «parecer» pour rédiger avec succès les leçons sur les saints ${ }^{65}$. Soulignant, pour commencer, la différence entre l'écriture des vies des saints et de celle des leçons pour les matines, Morales indique «que tout ce qui sera dit doit l'être de façon brève, simple et mesurée dans une certaine limite ${ }^{66}$. La proposition est soulignée dans le manuscrit et annotée d'un « dize muy bien » de la part de Philippe II, bien au fait du rapport entre clarté du discours et dévotion, comme il le souligne plus loin dans le texte en prenant l'exemple des miracles: «les miracles vérifiés et racontés avec simplicité et dans les temps édifient beaucoup et provoquent la dévotion ». Même si «la pieuse dévotion et la bonne occasion d'enseigner dans les leçons » exigent un dicours plus nourri, les leçons devront toujours être propres à «enseigner les âmes et les élever et les enflammer avec dévotion » sans qu'il soit nécessaire « de dire des choses effrayantes sur les miracles ».

Morales se montre soucieux de respecter les décrets tridentins et d'en suivre l'exemple donné par Rome, faisant confiance aux «hommes doctes qui composèrent les leçons du bréviaire romain couramment utilisé pour les prières et il serait bien de cette façon de tirer quelques éléments et d'imiter le style de presque toutes [ces leçons] ${ }^{67}$. Néanmoins, Philippe

\footnotetext{
${ }^{62}$ Session V, 17 juin 1546.

${ }^{63}$ Les théoriciens de la chaire, au XVIe siècle, recommandaient l'étude des orateurs de l'Antiquité : ÉRASME, dans l'Ecclesiastes, Louis de GRENADE, dans Ecclesiasticae Rhetoricae en 1576. Charles DEJOB a dénombré seize titres de traités sur le perfectionnement de la chaire entre 1562 et 1601, dans De l'influence du concile de Trente..., op. cit., p. 115.

${ }^{64}$ U. LANGER, Vertu du discours, Discours de la vertu, Droz, Seuils de la Modernité, 1997.

65 Ambrosio de MORALES, Copia de los apuntamientos que tubo Ambrosio de Morales sobre lo de los liçionarios para la orden de Su Magestad y de la repuesta que Su Magestad mando hazer a ellos. En Março 1566, «Lo que paresce se deve advertir para hazer con acertamiento las leciones de los sanctos es... », AGS, Casas y Sitios Reales, dossier 258, fol. 225.

${ }^{66}$ Ibid. : «que todo lo que se dixere [des lectiones] ha de ser breve abierto y sencillo y tasado con una notable limitación ».

${ }^{67}$ Ibid. : «los milagros averiguados y contados con llaneza y a sus tiempos mucho hedifican y provocan a devoción »; «la devoción piadosa y la buena ocassión de enseñar en las leçiones »; « enseñar las almas y
} 
II, soucieux de respecter le concile, ne favorise pas forcément l'imitation d'un modèle romain et semble plutôt fidèle au bréviaire de l'ordre hiéronymite dans son ensemble, suggérant seulement de remplacer quelques leçons par d'autres «qui possèdent les qualités nécessaires ». L'application des décrets conciliaires était une priorité du roi pour l'organisation de son monastère et de son église mais sans pour autant bousculer la tradition, laissant ainsi place à un prudent compromis entre les nouvelles règles et les anciennes qui avaient su maintenir la dévotion. José de Sigüenza, en observateur enthousiaste, sublime l'art de l'Escorial qui est capable d'élever les âmes sur son modèle d'harmonie et de symétrie. L'éloge est aussi pour Philippe II, initiateur de cette « octava maravilla : : ce Prince nous rendit à l'évidence, et fit en sorte que nous comprîmes la raison que possèdent les arts euxmêmes, et celle de l'harmonie dans laquelle ils agissent sur nos âmes ${ }^{68}{ }^{»}$. Le programme des saints, à l'Escorial, rend compte non seulement une nouvelle fois de l'implication du roi dans l'organisation de la vie religieuse de son Palais-monastère mais également des réflexions sur l'art comme parole visuelle agissant sur les âmes de façon comparable aux vibrants sermons d'une catholicité militante et conquérante.

levantarlas y ençenderlas con devoçion »; « de dezir cossas espantosas con milagros »; « hombres doctos que compusieron las leçiones del brivarío romano que agora comunmente rrecamos y assi sera bien tomar de ally algunas cossas y ymitar aquel estilo cassi en todas »; «que tengan las qualidades necessarias ».

${ }^{68}$ José de SIGÜENZA, op. cit., p. 597 : «este Principe nos puso en razon, y nos hizo que adviertessemos a la que las artes tienen en si mismas, y a la proporción que hazen con nuestras almas ». 